\title{
Red Herrings: Some Thoughts on the Meaning of Zero-Probability Events and Mathematical Modeling
}

By

\section{Edi Karni*}

\begin{abstract}
Kicking off the discussion following Savage's presentation at the 1952 Paris colloquium, Arrow raised what he considered to be a difficulty with the intuitive interpretation of Savage's theorem. It suggests that decision makers strictly prefer betting on an event of measure zero over betting on a proper subset of that event. Within the realm of the revealed-preference methodology and limited verifiability, Arrow's difficulty is a red herring: the problem he poses has it origin in technical aspects of the Savage's model and not in the substantive aspect of it.
\end{abstract}

Keywords: Zero-probability events, Null events, revealed preference

JEL Classification: D8

* Department of Economics, Johns Hopkins University. E-mail address karni@jhu.edu. I benefited from discussions and comments with Itzhak Gilboa, Joseph Greenberg, Dov Samet, Marco Scarsini, and David Schmeidler. None of them is responsible for or necessarily shares the views expressed in this note. 


\section{Arrow's Difficulty}

In May 1952, during the celebrated Paris colloquium, Savage presented his subjective expected utility theory. Following the presentation, Arrow raised what he considered to be a difficulty with the intuitive interpretation of Savage's theorem (see [1]). Arrow's point may be paraphrased as follows: A point is to be selected at random from a uniform distribution on the unit square. ${ }^{1}$ Let the event $A$ consist of all the points on the two diagonals and the event $B$ be their intersection. A decision maker whose beliefs are represented by the implied subjective probability measure assigns each of the two events zero probability and should, therefore, be indifferent between winning a prize if $A$ obtains and winning the same prize if $B$ obtains. Yet evidently, according to Arrow, presented with such choice decision makers would strictly prefer winning the prize in the event $A$ over winning in the event $B$.

The intuition articulated by this example is that since $B$ is a proper subset of $A$, betting on it must be less attractive than betting on $A$. This intuition is consistent with Savage's theory provided that $A$ is nonnull. ${ }^{2}$ If $A$ is null, the intuition fails. If Arrow's claim regarding the preferences of decision makers is correct, then the event $A$ is nonnull, which is inconsistent with the nonatomicity of the measure. ${ }^{3}$

Following Arrow's remarks, and recognizing the difficulty he raised as real, Frechet suggested that the problem may be avoided if a theory is developed of ranking of probabilities on events that are now considered to be null. ${ }^{4}$

Within the realm of the revealed preference methodology, Arrow's criticism is inadmissible - not because the events underlying the bets being compared are of probability measure zero but because these events are nonverifiable. Thus, asking a decision maker to express his preferences between

\footnotetext{
${ }^{1}$ The unit square should be thought of as Savage's state-space, the uniform distrbution is the subjective probability. The same point applies to the von Neumann-Morgenstern expected utility theory, in which the density function is objectively given.

${ }^{2} \mathrm{An}$ event $E$ is null if the decision maker is indifferent among all acts that agree on the complement of $E$. It is nonnull if it is not null.

${ }^{3}$ To see this, let $x \succ y$ and consider the act, $x_{E} y$, whose payoff is $x$ if the event $E$ obtains and $y$ if $E$ does not obtain. Since $B \subset A$ if $B$ is null, $y \sim x_{B} y$. If $x_{A} y \succ x_{B} y$ then, by transitivity, $x_{A} y \sim y_{A} y$, and $A$ is nonnull.

${ }^{4}$ Blume, L., Brandenburger, A., and E. Dekel [2], axiomatized a non-Archimedean subjective expected utility model which accomodates the possibility of conditioning choice on events of measure zero.
} 
betting on event $A$ and betting on event $B$ is a hypothetical choice question that has no meaning in the context of revealed-preference theory.

The issue here is not specific to the example at hand; it has some bearing on the use, and abuse, of mathematics in economics theory. Clearly, the use of infinity and limits in modeling economic behavior is an idealization, allowing the application of mathematical reasoning that facilitates the analysis of complicated models. A byproduct of the "mathematization" of economic analysis, however, is that the models incorporate mathematical constructs that have no empirical counterparts. Taking all the aspects of a model seriously, forces one to look for such empirical counterparts and, in the process, come up with red herrings (that is, problems involving the empirical interpretation of concepts and relations that are "in the model" but have no real-world meaning). Trying to render these mathematical constructs empirical sense is a fruitless effort.

\section{Impossible Events and Unverifiable Events}

Consider a finite state space example in which a die of six faces, marked by the numbers 1 through 6 , is rolled. The event that the die lands on a face marked by the numbers 7 or 8 is logically impossible to obtain. ${ }^{5}$ That is, given the information, it is logically impossible that the true state, which is one of the numbers 1 through 6 , is in the set $\{7,8\}$ or in any subset of it.

Blume, Brandenburger, and Dekel [2] gives an example in which the state space includes the edges and the corners of the die. These events have firstorder zero probability because, presumably, they are physically (rather than logically) impossible to obtain.

These examples are essentially different from the case of the event $A$ described above. Unlike the event $\{7,8\}$, event $A$ is not logically impossible. Furhtermore, the problem presented by $A$ is not whether it is physically possible to obtain. From a revealed-preference point of view, it is legitimate to ask the decision maker whether he prefers betting on the event $\{7,8\}$ or on the event $\{7\}$ because, after rolling the die, it is possible to verify the true state. The same is true with respect to the state space in the example of Blume, Brandenburger and Dekel (1991) that includes the edges

\footnotetext{
${ }^{5} \mathrm{An}$ event is said to obtain if it contains the true state.
} 
and the corners of the die. The event that the die lands on one of its edges is observable, hence, verifiable.

In contrast to these cases, the experiment described by Arrow is not implementable. Drawing a pair of real numbers in the unit square according to a nonatomic measure is a mathematical idealization. Any real-life chance device may only approximate (e.g., by a finite decimal expansion) these numbers. Yet consideration of betting on events such as $A$ and $B$ requires that the exact pair of real numbers be determined. According to revealed-preference methodology, a choice between acts that agree outside such events is strictly hypothetical and, consequently, inadmissible. Put differently, since states represent resolutions of uncertainty, it impossible to attain the complete resolution of the uncertainty as required by the formal model. Reality admits only partial resolution of uncertainty, which renders some mathematicallydistinct bets observationally equivalent. ${ }^{6}$

The following example may help clarify the issue of nonverifiability of conceivable events. Suppose the decision maker is asked to choose between betting on the event that the velocity of a particle in a given position takes one of a finite numbers of values and betting on it being one of these values. This question is inadmissible in the framework of the revealed preference methodology. ${ }^{7}$

\section{Conclusion}

Suppose that a decision maker faced with the choice of betting on $A$ or $B$ chooses, as Arrow predicted, to bet on $A$, even if the payoff is slightly smaller. Further yet, suppose that he is willing to pay something for the right to participate in the bet on $A$. What conclusions must be drawn from these choices?

The obvious conclusion is that the decision maker does not understand the problem. This is a clear-cut case in which the mathematical structure of the

\footnotetext{
${ }^{6}$ In the context of games, this observations means that if the set of pure strategies has the power of the continuum, it is impossible to implement a mixed strategy according to which the pure strategy is selected randomly according to a density function.

${ }^{7}$ According to the Heisenberg uncertainty principle, it is impossible to measure simultaneously the position and the velocity of a particle with any degree of accuracy or certainty. Consequently, it is impossible to determine the payoff of the bet.
} 
model allows the formulation of questions that have no empirical substance. Posing such a question in the first place is misleading. Taking the decision maker's response to such questions seriously as a test of Savage's theory is misguided.

The choice question should be rephrased as follows. How much are you be willing to pay to participate in a bet on $A$ given that, because it is impossible to find out if $A$ obtained, you can never win? I conjecture that the answer will be "nothing", which is consistent with treating $A$ as a null event.

\section{References}

[1] Colloques Internationaux du Centre National de la Research Sceintific, XL, Econometrie, (1953).

[2] Blume, L., Brandenburger, A., and E. Dekel (1991) "Lexicographic Probabilities and Choice Under Uncertainty," Econometrica. 